\title{
Excessive Treadmill Training Produces different Cardiac-related MicroRNA Profiles in the Left and Right Ventricles in Mice
}

\section{(우(1) $(8)$}

\author{
Authors

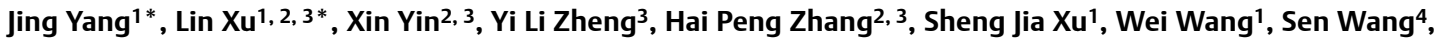 \\ Chen Yu Zhang ${ }^{3}$, ji Zheng $\mathrm{Ma}^{1}$
}

Affiliations

1 The Research Center of Military Exercise Science, The Army Engineering University of PLA, Nanjing, China

2 Department of Exercise and Heath, Nanjing Sport Institute, Nanjing, China

3 State Key Laboratory of Pharmaceutical Biotechnology, Jiangsu Engineering Research Center for MicroRNA Biology and Biotechnology, School of Life Sciences, Nanjing University, Nanjing, China

4 Department of Geriatric Cardiology, First Affiliated Hospital of Nanjing Medical University, Nanjing, China

Key words

excessive training, aerobic training, myocardial, microRNAs

accepted 17.06.2021

published online $\quad 20.08 .2021$

Bibliography

Int J Sports Med 2022; 43: 219-229

DOI 10.1055/a-1539-6702

ISSN $\quad 0172-4622$

2021. The Author(s).

This is an open access article published by Thieme under the terms of the Creative Commons Attribution-NonDerivative-NonCommercial-License, permitting copying and reproduction so long as the original work is given appropriate credit. Contents may not be used for commercial purposes, or adapted, remixed, transformed or built upon. (https://creativecommons. org/licenses/by-nc-nd/4.0/)

Georg Thieme Verlag KG, Rüdigerstraße 14,

70469 Stuttgart, Germany

Correspondence

Prof. Ji Zheng Ma

The Research Center of Military Exercise Science,

The Army Engineering University of PLA

60 Shuang Long Jie Road

Nanjing

211101 jiangsu

China

Tel.: 86-25-83686234, Fax: 86-25-83686234

mjz_mjj@sina.com

These two authors contributed to the work equally and should be regarded as co-first authors.
Chen Yu Zhang

School of life sciences,

Nanjing University

168 Xian Lin Road

Nanjing,

210023 Jiangsu

China

Tel.: 86-25-89681276

cyzhang@nju.edu.cn

Sen Wang

Department of Geriatric Cardiology,

First Affiliated Hospital of Nanjing Medical

University, 300 Guangzhou Road

Nanjing

210029 Jiangsu

China

Tel.: 86-25-83718836

44384587@qq.com

Supplementary Material is available under https://doi.org/10.1055/a-1539-6702

\section{ABSTRACT}

High-volume training followed by inadequate recovery may cause overtraining. This process may undermine the protective effect of regular exercise on the cardiovascular system and may increase the risk of pathological cardiac remodelling. We evaluated whether chronic overtraining changes cardiac-related microRNA profiles in the left and right ventricles. C57BL/6 mice were divided into the control, normal training, and overtrained by running without inclination, uphill running or downhill running groups. After an 8-week treadmill training protocol, the incremental load test and training volume results showed that the model had been successfully established. The qRT-PCR results showed increased cardiac miR-1, miR-133a, miR133b, miR-206, miR-208b and miR-499 levels in the left ventricle of the downhill running group compared with the left ventricle of the control group. Similarly, compared with the control group, the downhill running induced increased expression of miR-21, miR-17-3p, and miR-29b in the left ventricle. Unlike the changes in the left ventricle, no difference in the expression of the tested miRNAs was observed in the right ventricle. Briefly, our results indicated that overtraining generally affects key miRNAs in the left ventricle (rather than the right ventricle) and that changes in individual miRNAs may cause either adaptive or maladaptive remodelling with overtraining. 


\section{Introduction}

Overall, several physical exercises generate salutary effects on all physiological systems within the body [1]. Among parts of the body, the heart, the primary pumping organ of the circulatory system, powers human blood circulation and carries out many important functions [2]. Thus, aerobic fitness is well known to be protective for the cardiovascular system, but this protection can shift according to the type, intensity, frequency and duration of exercise [3]. The heart can become fit for chronic exercise by increasing the heart volume, the formation of new blood vessels, and reducing the collagen content to meet the oxygen requirements of the body [4]. Together, these effects are called "athlete's heart", which describes cardiac physiological remodelling that occurs in response to an increased workload [5]. Because these adaptations play a significant role in improving sports performance and fitness, the molecular mechanisms underlying exercise-induced cardiac remodelling, including a variety of signalling pathways and downstream mediators, in the myocardium have been recognized $[6,7]$.

Variations in training stimuli have been suggested to be necessary to optimize these adaptations [8]. The optimization of human performance is the primary objective of exercise training. High-intensity endurance training is needed to achieve a high level of performance. The widely known law of supercompensation is a key principle of physical training required for peak performance. Therefore, after proper training and recovery periods, a positive adaptation or performance improvement may occur. This process is called functional overreaching [9]. However, intense, high-volume training under conditions of insufficient recovery will increase the cumulative biological burden, eventually leading to breakdown of the internal balance and causing activation of the stress response system and long-term maladjustments in signs and symptoms [9]. This state can manifest as nonfunctional overreaching (NFOR) or overtraining (OT), which are on the same spectrum of states that may cause the development of overtraining syndrome (OTS), resulting in decreased performance and profound fatigue [9]. Although the mechanism of overtraining in endurance training is unclear, pathological cardiac remodelling has been observed in mice following excessive treadmill training [10].

The differential expression of microRNAs (miRNAs) in the heart is detected in the aerobic training adaptive response during/after exercise, which can alter gene expression via posttranscriptional adjustment and may also affect translational events, such as cardiomyocyte growth and proliferation [11-13]. Alterations in cardiac miRNAs (miR-1, miR-133a/b, miR-208a/b, and miR-499) that are abundantly expressed in the myocardium [14] have been implicated as one of the regulatory mechanisms of long-term adverse cardiac functional adaptation and remodelling [14]. In addition, other miRNAs, such as miR-206, miR-21, miR-17-3p, and miR-29b, have been reported to be involved in cardiac cellular reprogramming in response to aerobic exercise [13]. Thus, aerobic training promotes heart health. However, long-term excessive exercise may increase the risk of pathological heart remodelling. Pathological cardiac remodelling is usually a response to pathological conditions such as hypertension, heart disease, and aortic stenosis. It mainly manifests as eccentric hypertrophy $[15,16]$, an increasing degree of fibrosis [17, 18] and cardiac amyloidosis [19]. Interestingly, similar pathological adaptations were also observed in overtrained mice, including left ventricle (LV) pathological hypertrophy, signs of fibrosis and negative morphological adaptations [10]. At present, the miRNAs affected by excessive training remain unclear. Furthermore, the right ventricle (RV) differs from the LV in many fundamental ways, and the two chambers differ in their morphology and metabolism [20]. The RV is connected to the low-pressure pulmonary vascular system, which is accompanied by low vascular resistance, while the $\mathrm{LV}$ is exposed to higher pressure conditions with high vascular resistance [20] . Currently, the difference between $\mathrm{LV}$ and $\mathrm{RV}$ after overtraining has not been studied. More importantly, mouse overtraining models have been developed to elucidate the effects of different training regimes on numerous structures and functions of the body [21]. In the present study, changes in cardiac miRNAs in response to three treadmill running OT models were evaluated in mice, and whether chronic excessive exercise results in differential responses in the RV and LV was examined.

We hypothesized that OT affects these cardiac miRNAs and that changes to the two ventricles differ, providing a reference for understanding the physiological response to different overtraining protocols and determining the best training strategy for optimal performance.

\section{Materials and Methods}

\section{Experimental animals}

A total of 80 male C57BL/6 J mice ( 8 weeks old) were obtained from Beijing Vital Laboratory Animal Technology Co., Ltd. (Beijing, China). Twenty cages housing 4 mice each were placed in a temperature-controlled room $\left(22 \pm 2^{\circ} \mathrm{C}\right)$ with a 12 -h light/dark cycle (light: 9 AM to 9 PM, dark: 9 PM to 9 AM) and unlimited water and food. The experimental procedures were approved by the Nanjing University Animal Care and Use Committee. This protocol met the ethical standards of the International Journal of Sports Medicine [22]. Eight-week-old C57BL/6 mice were divided into 5 groups: (1) control (CON; sedentary mice; $n=16) ;(2)$ normal training (NT; trained with adequate recovery, $n=16$ ); (3) overtrained by running without inclination (OTR; an OT protocol based on running without inclination; $n=16$ ); (4) overtrained by uphill running (OTR/up; an OT protocol based on uphill running; $n=16)$; and (5) overtrained by downhill running (OTR/down; an OT protocol based on downhill running; $n=16$ ) groups. The groups were trained or manipulated in a lit room between 1 PM and 5 PM.

\section{Performance evaluations Incremental load test (ILT)}

The mice were exercised on a treadmill (ZH-PT, Zhenghua, Anhui, China) with an inclination of $0 \%$ at a speed of $10 \mathrm{~m} / \mathrm{min}$ for $10 \mathrm{~min}$ and continued to adapt for 5 days. As described by Pereira BC et al., the mice were subjected to the ILT [21]. The initial inclination of the treadmill was $0 \%$, the initial intensity was $6 \mathrm{~m} / \mathrm{min}$, and then, the speed was increased by $3 \mathrm{~m} / \mathrm{min}$ every $3 \mathrm{~min}$ until the mice were exhausted (exhaustion standard: mice touched the end of the treadmill 5 times within $1 \mathrm{~min}$.). We used physical stimulation to encourage the mice to exercise. If a mouse became exhausted without completing a phase, the exhaustion velocity $(E V=V+(n / b) \times a$, where $V$ is the velocity of the last completed phase, $n$ is the dura- 
tion maintained in the incomplete phase, $b$ is the duration of the phase, and $a$ is the test enhancement) was calculated using the methods described by Kuipers et al. [23]. The EV of the mice was used to determine the intensity of the aerobic training and overtraining protocols.

\section{Rotarod test}

We used a rotarod treadmill (JLBehv-RRTM, Jiliang, Shanghai, China) to evaluate the coordination and balance capabilities of rodents. The initial speed of the mice on the rotarod treadmill was $3.5 \mathrm{rpm}$, and then the speed increased evenly and reached $35 \mathrm{rpm}$ after $300 \mathrm{~s}$. The time the mice stayed on the top of the rotarod treadmill was recorded, and three consecutive experiments were performed on each mouse to record its best performance [24].

\section{OT protocols, aerobic training protocol and performance evaluation}

Briefly, this experiment was performed according to the 8-week OT protocol with different inclination angles as previously described by Pereira BC et al. [21]. The mice received 5 days of training and 2 days of recovery during the experimental week ( $\triangleright$ Table 1 ). In the first 4 weeks, rodents ran at an inclination of $0 \%$. From week 5 of OT, the rodents ran at an inclination of $0 \%$ (OTR), $14 \%$ (OTR/up) or-14\% (OTR/down). These running grades were sustained until the end of the OT protocols. During the experiment, we observed and recorded the movement of the mice every day. The OT groups (including OTR, OTR/up and OTR/down) were unable to complete the prescribed training load beginning in the sixth week. We recorded the exercise time of these rodents (exhaustion standard: mice touched the end of the treadmill 5 times in $1 \mathrm{~min}$ ), and mice in the NT group were able to complete the training according to the established training volume. Therefore, we recorded the training volume ( $\mathrm{min}$ ) in the OT groups from the sixth week. The NT group used in the present study adopted an 8-week aerobic training protocol without inclination ( $>$ Table 2 ). The mice received 5 days of training and 2 days of recovery during the experimental week.

The ILT test was conducted in week 0 and $48 \mathrm{~h}$ after the last OT sessions at the ends of weeks 4 and 8 . The rotarod test was performed only at the end of the 4th and 8th weeks of training. In week 0 , all groups (the CON, NT, OTR, OTR/up and OTR/down groups) carried out the ILT without inclination. However, $48 \mathrm{~h}$ after the end of weeks 4 and 8 , the CON, NT and OTR groups carried out the ILT without inclination, the OTR/up group carried out the ILT while running uphill, and the OTR/ down group carried out the ILT while running downhill. Four hours after the rotarod test, mice performed the ILT [21].

\section{Extraction of myocardial samples}

All mice were euthanized by cervical dislocation $48 \mathrm{~h}$ after the last ILT. Under sterile RNase-free conditions, the LV myocardium and $\mathrm{RV}$ myocardium were quickly frozen in liquid nitrogen and stored at $-80^{\circ} \mathrm{C}$ until the experimental analysis.

- Table 1 Characteristics of the overtraining protocols used in the present study.

\begin{tabular}{|l|l|l|l|l|l|l|l|l|}
\hline Week & $\begin{array}{l}\text { Intensity } \\
\text { (\% EV) }\end{array}$ & $\begin{array}{l}\text { Duration } \\
\text { (min) }\end{array}$ & \multicolumn{2}{|c|}{ Daily sessions } & $\begin{array}{l}\text { Recovery between } \\
\text { sessions (h) }\end{array}$ & $\begin{array}{l}\text { TR (\%) } \\
\text { OTR }\end{array}$ & $\begin{array}{l}\text { TR (\%) OTR/ } \\
\text { up }\end{array}$ & $\begin{array}{l}\text { TR (\%) OTR/ } \\
\text { down }\end{array}$ \\
\hline 1 & 60 & 15 & 1 & & 24 & 0 & 0 & 0 \\
\hline 2 & 60 & 30 & 1 & & 24 & 0 & 0 & 0 \\
\hline 3 & 60 & 45 & 1 & & 24 & 0 & 0 & 0 \\
\hline 4 & 60 & 60 & 1 & & 24 & 0 & 0 & 0 \\
\hline 5 & 60 & 60 & 1 & & 24 & 0 & 14 & -14 \\
\hline 6 & 70 & 60 & 1 & & 24 & 0 & 14 & -14 \\
\hline 7 & 75 & 75 & 1 & & 24 & 0 & 14 & -14 \\
\hline 8 & 75 & 75 & 2 & & 4 & 0 & 14 \\
\hline
\end{tabular}

TR: Treadmill grade, OTR: OT protocol based on running without inclination, OTR/up: OT protocol based on uphill running, OTR/down: OT protocol based on downhill running.

- Table 2 Characteristics of the aerobic training protocol used in the present study.

\begin{tabular}{|c|c|c|c|c|c|}
\hline Week & Intensity(\%EV) & Duration(min) & Daily session & $\begin{array}{c}\text { Recovery between } \\
\text { sessions(h) }\end{array}$ & Treadmill grade( \%) \\
\hline 1 & 60 & 15 & 1 & 24 & 0 \\
\hline 2 & 60 & 30 & 1 & 24 & 0 \\
\hline 3 & 60 & 45 & 1 & 24 & 0 \\
\hline 4 & 60 & 60 & 1 & 24 & 0 \\
\hline 5 & 60 & 60 & 1 & 24 & 0 \\
\hline 6 & 60 & 60 & 1 & 24 & 0 \\
\hline 7 & 60 & 60 & 1 & 24 & 0 \\
\hline 8 & 60 & 60 & 1 & 24 & 0 \\
\hline
\end{tabular}




\section{RNA isolation and quantification of miRNAs}

For total RNA isolation, the frozen ventricular myocardium was homogenized using RNAiso Plus (Code No. 9109, Takara, Dalian, China) according to the corresponding protocol. After all samples were processed in the same batch, real-time quantitative polymerase chain reaction (RT-qPCR) was used to measure the abundance of miRNAs in myocardial tissue. Nanodrop ND 1000 (Thermo Scientific, Wilmington, USA) was used to measure the RNA concentration and quality (OD260/280 ratio). The hydrolysis probe for RTqPCR was used to generate the corresponding cDNA product, and the TaqMan PCR kit (Thermo Fisher Scientific, Waltham, United States) was used with a Roche LightCycler®96 (Roche, Basel, Switzerland). The real-time PCR conditions consisted of a preincubation step at $95^{\circ} \mathrm{C}$ for $5 \mathrm{~min}, 40$ cycles of $95^{\circ} \mathrm{C}$ for $15 \mathrm{~s}$ and $60^{\circ} \mathrm{C}$ for $60 \mathrm{~s}$, and finally cooling at $37^{\circ} \mathrm{C}$ for $30 \mathrm{~s}$. The following TaqMan ${ }^{\circledR}$ miRNA assays were used: snRNA U6 (ID: 001973), hsa-miR-1 (ID: 002222), mmu-miR-17-3p (ID: 002543), hsa-miR-21 (ID: 000397), hsa-miR-29b (ID: 000413), hsa-miR-133a (ID: 002246), hsa-miR133b (ID: 002247), hsa-miR-208 (ID: 000511), hsa-miR-208b (ID: 002290), hsa-miR-206 (ID: 000510) and mmu-miR-499 (ID: 001352). The relative expression of miRNAs in the myocardium was normalized to endogenous U6 snRNA expression in the tissue, $\mathrm{Ct}$ values were calculated using the $2^{-\Delta \Delta C t}$ method, and each sample was analysed three times.

\section{H\&E staining and Masson staining}

Myocardial tissue was fixed with $4 \%$ paraformaldehyde for $24 \mathrm{~h}$ (G1101, Servicebio, Wuhan, China), dehydrated using an automatic dehydrator (Donatello, DIAPATH, Milan, Italy) for $16 \mathrm{~h}$, and then inserted into a paraffin embedding machine (JB-P5, Wuhan Junjie Electronics Co., Ltd., Wuhan, China) to prepare sections.

\section{H\&E staining}

We immersed the paraffin sections in xylene (I) and (II) (10023418, Chemical Reagent Sinopharm Holding Co., Ltd., Beijing, China) for 20 min for dewaxing. Then, the samples were soaked in a gradient of alcohol solutions (100092683, Sinopharm Chemical Reagent Co. Ltd., Beijing, China) for 5 min and rinsed with running water. Haematoxylin staining solution (G1003, Servicebio, Wuhan, China) was used to stain the tissue sections for 5 min before they were rinsed thoroughly with running water and immersed in the differentiation solution for $30 \mathrm{~s}$. After the slices were dehydrated and rendered transparent, they were sealed with neutral gum (10004160, Sinopharm Chemical Reagent Co. Ltd, Beijing, China), observed and images were captured under an optical microscope.

\section{Masson staining}

The paraffin sections were dewaxed in a xylene solution (10023418, Sinopharm Chemical Reagent Co. Ltd, Beijing, China) and then soaked in Masson's pine solution (G1006, Servicebio, Wuhan, China) overnight. The sections were incubated in the dye solution composed of an equal ration of Masson's B solution and Masson's $C$ solution for $1 \mathrm{~min}$, and then differentiated with $1 \%$ hydrochloric acid alcohol. The slices were soaked in Masson's pine D solution and Masson's pine E solution for $6 \mathrm{~min}$ and $1 \mathrm{~min}$, respectively. After immersion in different solutions, all samples were rinsed with running water. The samples were dried and directly immersed in Mas- son's F solution for $30 \mathrm{~s}$. The sections were then rinsed with $1 \%$ glacial acetic acid for differentiation and dehydrated with anhydrous ethanol 3 times. Xylene was used to clear the sections for $5 \mathrm{~min}$, and the sections were sealed with neutral gum (10004160, Sinopharm Chemical Reagent Co. Ltd, Beijing, China), observed and images were captured under an optical microscope (NIKON ECLIPSE E100, Nikon, Tokyo, Japan). We selected the same position of the ventricle in each slice from the sample to capture images. Under the microscope, the nucleus and collagen fibres were stained blue. We used Image] [25] software to analyse the images and quantitatively calculate the area percentage of collagen fibres.

\section{Statistical analyses}

The results are reported as the means \pm standard errors of the means (SEM). GraphPad Prism 7 software was used for statistical analyses of all data generated in the experiments. In addition, twoway analysis of variance (ANOVA) was used to examine the effects on training volume and exhaustion velocity. One-way ANOVA was used to examine changes in body weight and the qRT-PCR results. The normality of the data distribution was tested using the Shapiro-Wilk W-test. In addition, the nonparametric Kruskal-Wallis test was used for values that did not exhibit a normal distribution. The significance level was set to $P<0.05$.

\section{Results}

\section{Body weight}

- Fig. 1a presents the change in body weight $(\mathrm{g}$ ) of the five groups during the experimental period. From the 5 th to the 8th weeks, the body weight of the NT group was significantly lower than that of the CON group $(P=0.0082, P=0.0014, P=0.0021$, and $P=0.0001)$. At week 8 , the weights of the OTR group, OTR/up and OTR/down groups were lower than that of the CON group $(P=0.0014$, $\mathrm{P}=0.0001$, and $\mathrm{P}=0.0010$, respectively). As indicated in $>\mathbf{F i g . ~ 1 b}$, the body weights of the NT group $(5.30 \pm 6.15 \%, P<0.0001)$, OTR group (2.46 $\pm 6.54 \%, P<0.0001)$, OTR/up group $(3.41 \pm 4.72 \%$, $\mathrm{P}<0.0001)$ and OTR/down group $(4.25 \pm 5.87 \%, \mathrm{P}<0.0001)$ were significantly lower than the body weight of the CON group $(16.18 \pm 5.14 \%)$ from weeks 0 to 8 . No significant difference in body weight was observed between the NT, OTR, OTR/up and OTR/down groups.

\section{Incremental load test (ILT)}

- Figure 2 a shows the differences in exhaustion velocity (EV) between the five groups. The EV in week 4 of the exercise groups was higher than the value recorded for this group in week 0 and the value of the CON group in week 4 (i. e., NT $=40.79 \%$ and $42.98 \%$, OTR $=15.92 \%$ and $29.61 \%$, respectively). The OTR/up and OTR/down groups had different ILT inclinations in week 4 , and thus they were not compared with the ILT result recorded in week 0 . Based on these results, 4 weeks of treadmill training improved exercise performance in mice in the experimental groups.

The EV recorded in week 8 for the OT groups was lower than the values recorded for these groups in week 4 (i. e., OTR $=-19.33 \%$, OTR/ up $=-10.91 \%$, and OTR/down $=-30.14 \%$ ), but the EV of the NT group was increased by $21.56 \%$. Compared with the EV at week 0 , the EV 
at week 8 was higher in the NT group (71.15\%), and other differences did not reach statistical significance. Thus, we successfully constructed overtraining models that were distinguished from normal endurance training models.

\section{Training volume}

- Figure $\mathbf{2 b}$ shows a significantly lower training volume measured in the first session in week 8 than that at week 6 for the OTR (i.e.,$23.30 \%$; $P<0.0001)$, OTR/up (-24.02\%; $P<0.0001)$, and OTR/down $(-29.13 \%$; $P<0.0001)$ groups, and the value was lower than that at week 7 for the three OT groups (i. e., OTR $=-16.08 \%, P=0.0116$;
OTR/up $=-15.33 \%, P=0.0202$ and OTR $/$ down $=-18.73 \%, P=$ $0.0034)$. Thus, the mice that carried out the OT protocols gradually accumulated an exercise load.

In addition, compared with the training volume measured in the first session of week 8 , the value measured in the second session of week 8 was lower for the OTR (20.30\%; P=0.0071), OTR/up (17.61\%, $\mathrm{P}=0.0245)$ and OTR/down (31.58\%, $\mathrm{P}<0.0001)$ groups. Similarly, the training volume recorded in the second session of week 8 was lower than that recorded at week 6 for the OTR $(-48.33 \%$; $\mathrm{P}<0.0001)$, OTR/up $(-45.86 \%$; $<<0.0001)$ and OTR/ down $(-69.90 \%$; $<<0.0001)$ groups and lower than that recorded

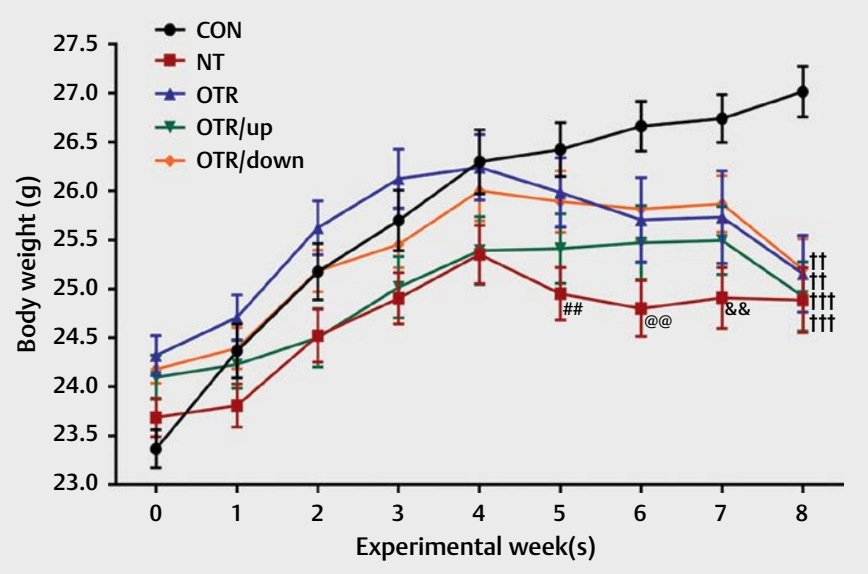

b

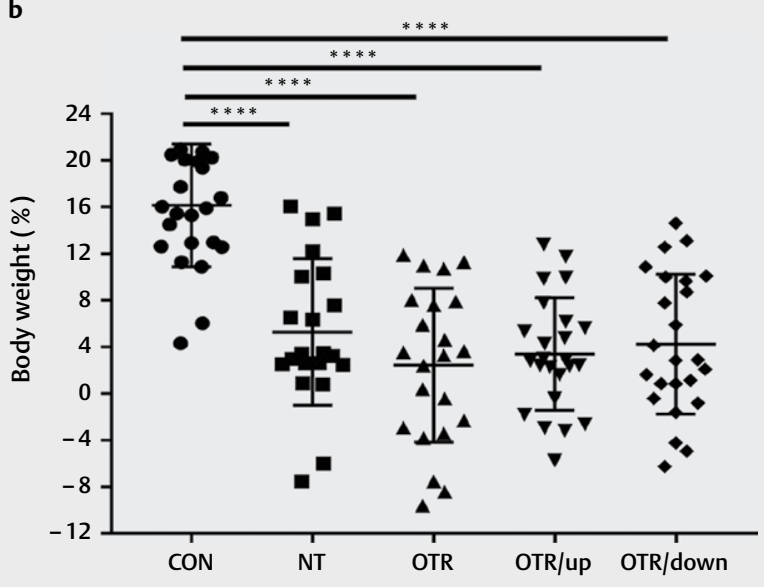

- Fig. 1 Changes in the body weight ( $\mathrm{g}$ ) of all experimental groups (a). Percent changes in body weight at the end of the 8-week treadmill protocol (b). The data represent the means $\pm S E M s$ of $n=16$. \#\# Statistically significant $(P<0.01)$ difference compared with the CON group in week 5; @@ statistically significant $(P<0.01)$ difference compared with the CON group in week 6 ; \&\&statistically significant $(P<0.01)$ difference compared with the CON group in week 7; \#statistically significant $(\mathrm{P}<0.01)$ difference compared with the CON group in week 8; \#istatistically significant $(\mathrm{P}<0.001)$ difference compared with the CON group in week $8 ;{ }^{* * * *}$ statistically significant $(\mathrm{P}<0.0001)$ difference compared with the CON group.
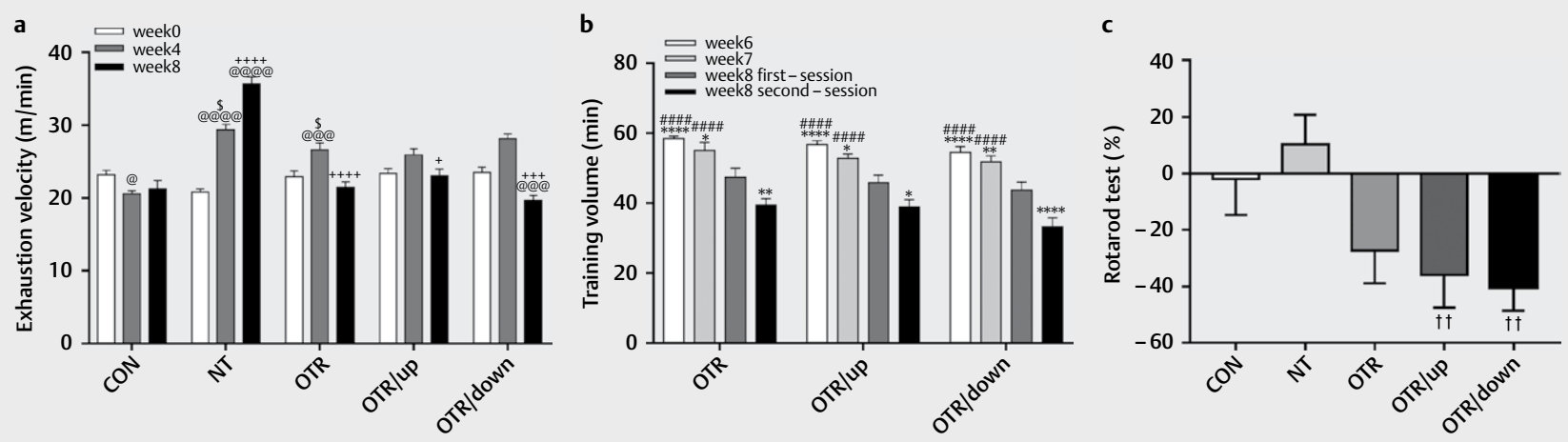

- Fig. 2 Changes in the exhaustion velocity $(\mathrm{m} / \mathrm{min}$ ) during the ILT at weeks 0,4 and 8 (a). The training volume $(\mathrm{min})$ is shown from the sixth to the eighth weeks (b). Percent alteration (\%) in the rotarod test between weeks 4 and 8 (c). The data represent the means \pm SEMs of $n=16$. ${ }^{\circledR}$ Statistically significant $(P<0.05)$ difference compared with week 0 ; @@@statistically significant $(P<0.001)$ difference compared with week 0; @@@@statistically significant $(P<0.0001)$ difference compared with week 0 ; ${ }^{+}$statistically significant $(P<0.05)$ difference compared with week $4 ;{ }^{++++}$statistically significant $(P<0.0001)$ difference compared with week 4; ${ }^{\text {s }}$ statistically significant $(P<0.05)$ difference compared with the CON group at week 4; ${ }^{*}$ stat istically significant $(P<0.05)$ difference compared with the first session in week $8 ;{ }^{*}$ " statistically significant $(P<0.01)$ difference compared with the

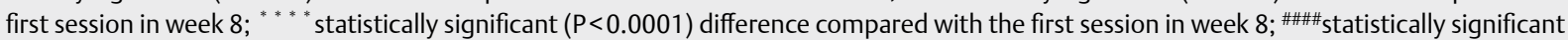
$(P<0.0001)$ difference compared with the second session in week 8 ; "istatistically significant $(P<0.05)$ difference compared with the NT group. 


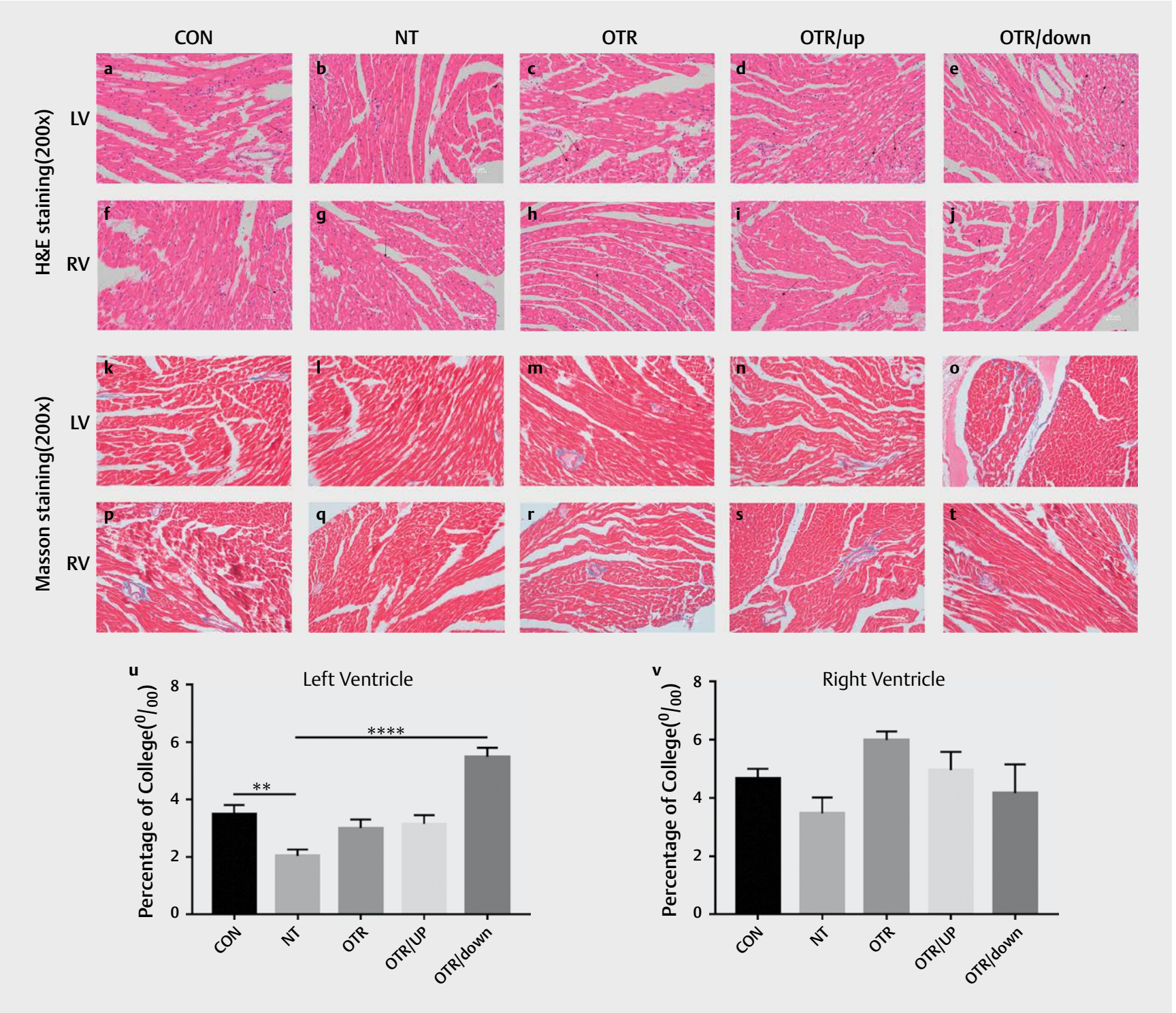

- Fig. 3 Representative images of H\&E staining (200x) of the left ventricular myocardium (a-e) and right ventricular myocardium ( $\mathbf{f}$-j) from the five experimental groups. Masson's trichrome staining (200x) of the left ventricular myocardium (k-o) and right ventricular myocardium (p-t) tissue sections from the five experimental groups. Scale bars represent $50 \mu \mathrm{m}$. Quantitative analysis of Masson's trichrome staining for fibrosis in the left ventricular myocardium (u). Quantitative analysis of Masson's trichrome staining for fibrosis in the right ventricular myocardium (v). The data represent the means \pm SEMs of $\mathrm{n}=6$. ${ }^{*} *$ Statistically significant $(\mathrm{P}<0.01) ;{ }^{* * * *}$ statistically significant $(\mathrm{P}<0.0001)$.

at week 7 for the three OT groups (i. e., OTR $=-39.64 \%, P<0.0001$; OTR/up $=-35.64 \%, P<0.0001$ and OTR/down $=-56.22 \%$, $\mathrm{P}<0.0001)$. Interestingly, although the accumulated load consistently increased, the training volumes measured in the same weeks did not differ between the three OT groups.

\section{Rotarod test}

- Figure 2c shows the percent alteration (\%) in the rotarod results from week 4 to week 8 . Compared with the NT group (10.39\%), the rotarod alteration was significantly higher than OTR/up (-35.85\%; $\mathrm{P}=0.0489)$ and OTR/down $(-40.28 \% ; \mathrm{P}=0.0159)$ groups. In addition, the results of the CON and OTR groups decreased by $-1.86 \%$ and-27.25\%, respectively, after 4 weeks of training, but the differences were not statistically significant.

\section{H\&E and Masson staining of LV and RV myocardium}

We investigated myocardial morphological changes by performing $\mathrm{H} \& \mathrm{E}$ staining and cardiac fibrosis using Masson's trichrome staining. As shown in > Fig. 3a-e, compared with the CON group, the number of disordered cells in the LV myocardium of the OTR/down group increased, the intercellular space increased and myocardial disarray occurred (marked by arrows in the figure), while no similar difference was observed when comparing the RV myocardium between groups ( $\vee \mathbf{F i g} \cdot \mathbf{3 f}-\mathbf{j}$ ). Compared with the CON group, col- 
a
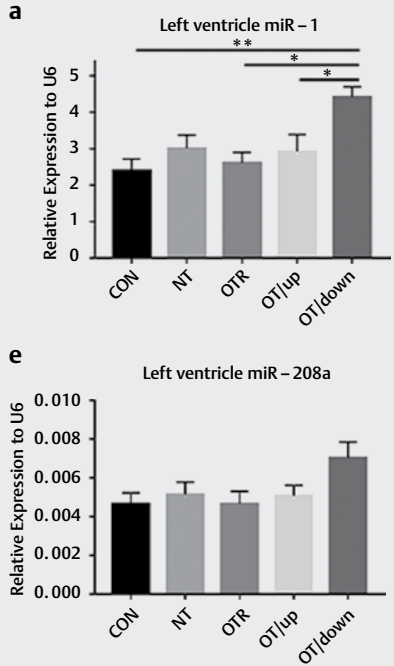

h
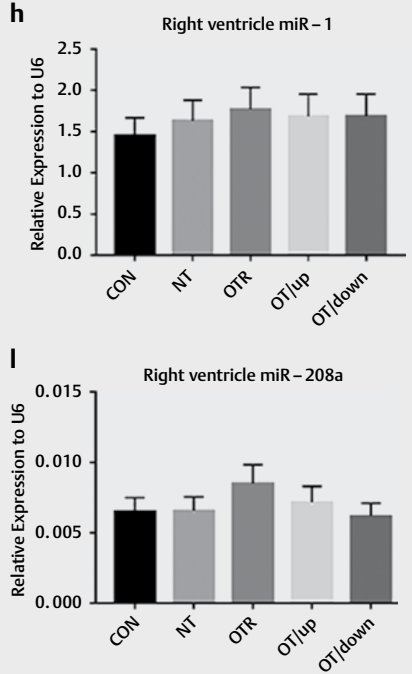

b

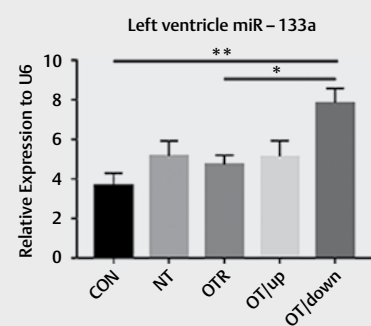

f

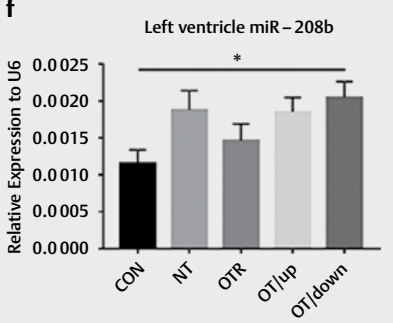

i

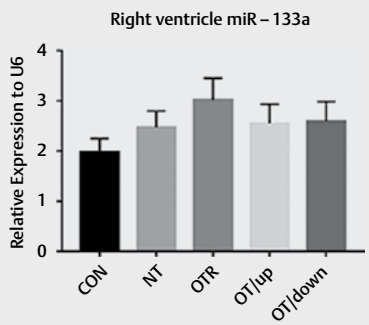

m

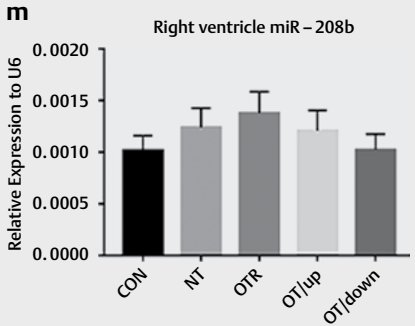

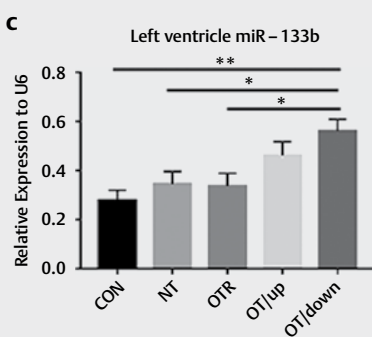

d
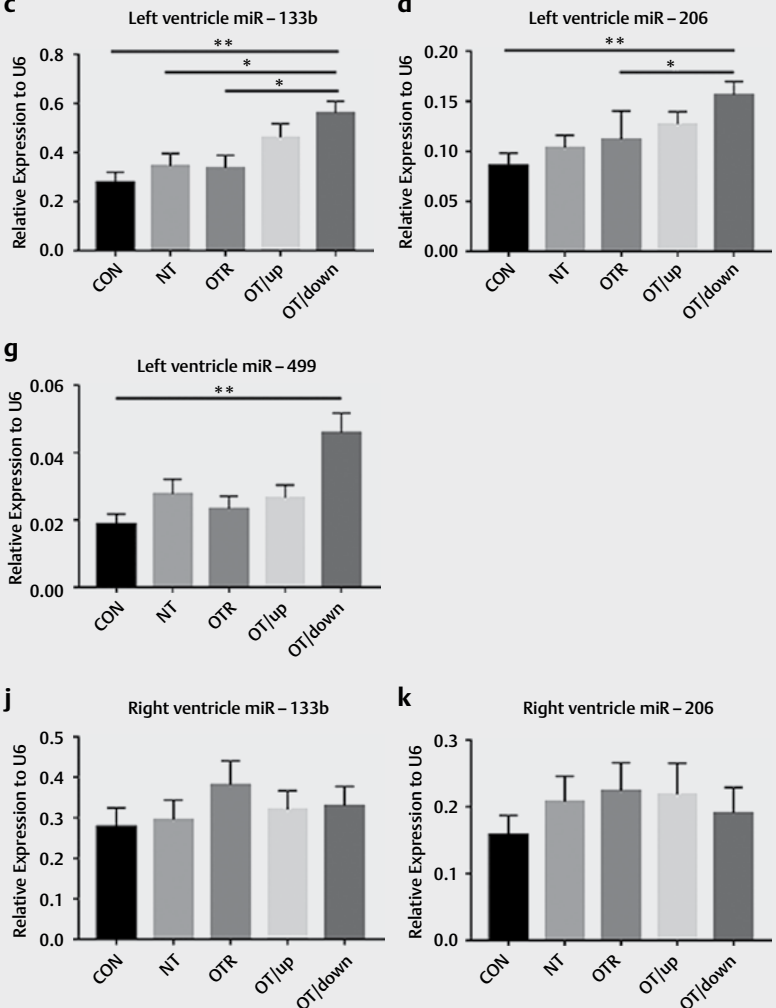

K Right ventricle miR -206

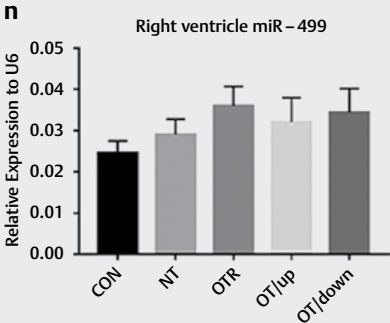

Fig. 4 Cardiac expression of miR-1 (a), miR-133a (b), miR-133b (c), miR-206 (d), miR-208a (e), miR-208b (f) and miR-499 (g) in the left ventricular myocardium of the five experimental groups. Cardiac expression of miR-1 (h), miR-133a (i), miR-133b (j), miR-206 (k), miR-208a (I), miR-208b (m) and miR-499 (n) in the right ventricular myocardium of the five experimental groups. The data represent the means \pm SEMs of $\mathrm{n}=16 .{ }^{*}$ Statistically significant $(\mathrm{P}<0.05) ;{ }^{*}$ " statistically significant $(\mathrm{P}<0.01)$.

lagen precipitation significantly decreased in the LV of the NT group after exercise training ( $\mathrm{P}=0.001)$; in contrast, the collagen precipitation in the LV tissue was significantly increased in the OTR/down group after downhill overtraining ( $P<0.0001$; $>$ Fig. 3k-o and $3 u)$. However, a similar difference did not appear in the RV ( $\vee$ Fig. 3p-T and $3 v$ ).

\section{Changes in cardiac miRNA levels in response to treadmill running}

As shown in $>$ Fig. 4, we examined the expression of cardiac miRNAs in the ventricular myocardium after the treadmill running protocol using qRT-PCR. An analysis of the LV myocardium revealed that the expression levels of miR-1, miR-133a, miR-133b, miR-206, miR-208b, and miR-499 in the OTR/down group were $\sim 1.84$-fold $(P=0.0026), \sim 2.14$-fold $(P=0.0015), \sim 2.02$-fold $(P=0.0013)$, $\sim 1.82$-fold $(\mathrm{P}=0.0035), \sim 1.77$-fold $(\mathrm{P}=0.0379)$, and $\sim 2.46$-fold $(P=0.0026)$ higher, respectively, than those in the CON group ( $\mathbf{F i g}$. 4a-d and $\mathbf{f}-\mathbf{g}$ ). The expression of miR-1, miR-133a, miR133b and miR-206 was increased $\sim 1.69$-fold $(P=0.0114), ~ 1.65$-fold $(P=0.0346), \sim 1.67$-fold $(P=0.0210)$, and $\sim 1.40$-fold $(P=0.0101)$, respectively, in the OTR/down group compared to the OTR group ( Fig. 4a-d). Moreover, the expression levels of miR-133b and miR-1 in the OTR/down group were $\sim 1.63$-fold $(P=0.0253)$ and $\sim 1.51$-fold $(P=0.0404)$ higher than those in the NT and OTR/up groups, respectively. In addition, we were surprised to find no similar changes in miRNA expression in the mouse RV myocardium ( Fig. 4h-n). Significant differences in the miRNA expression levels were not observed in the RV myocardium among the five experimental groups. Notably, no difference in miR-208a ( $>$ Fig. 4e) ex- 


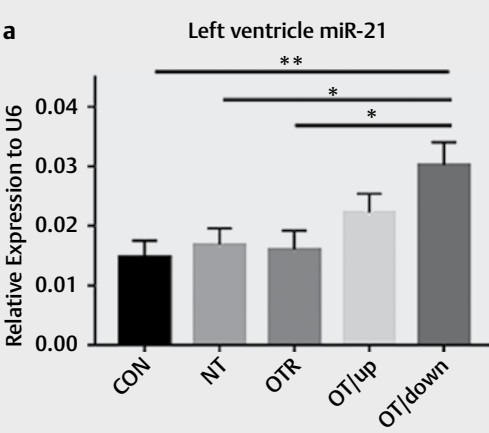

d

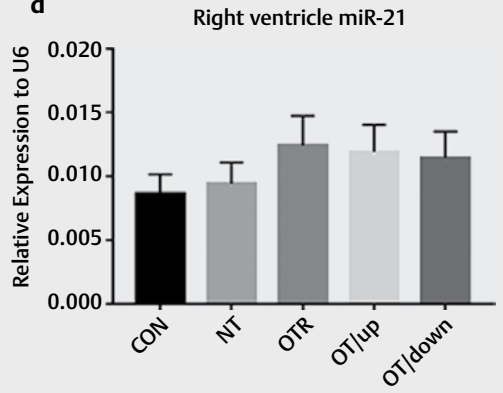

b

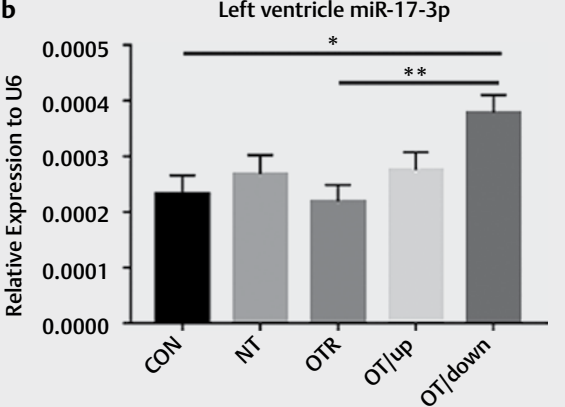

e

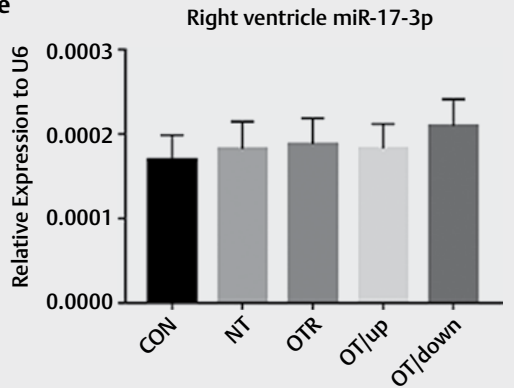

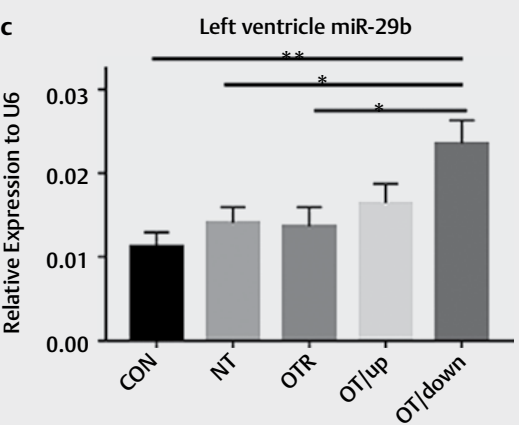

f

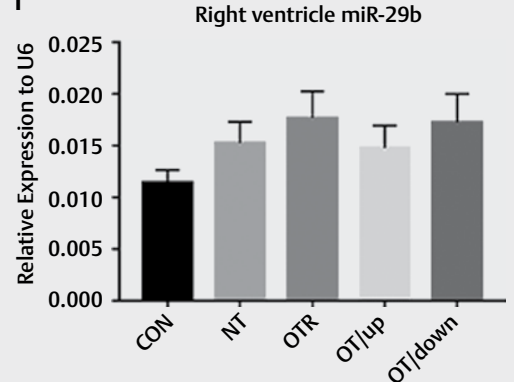

- Fig. 5 The expression of miR-21 (a), miR-17-3p (b), and miR-29b (c) in the left ventricular myocardium of the five experimental groups and miR-21 (d), miR-17-3p (e), and miR-29b (f) in the right ventricular myocardium of the five experimental groups. The data represent the means \pm SEMs of $n=16$. "Statistically significant $(P<0.05)$; " statistically significant $(P<0.01)$.

pression was detected between the LV myocardium and RV myocardium.

\section{Changes in other miRNA levels in response to the treadmill running protocol}

The expression levels of other miRNAs in ventricular samples from the experimental groups of mice were measured using qRT-PCR, and the results showed a similar phenomenon. Levels of miR-21, miR-17-3p and miR-29b in the LV myocardium were increased $\sim 2.05$-fold $(\mathrm{P}=0.0073), \sim 1.63$-fold $(\mathrm{P}=0.0178)$, and $\sim 2.09$-fold $(\mathrm{P}=0.0023)$, respectively, in the OTR/down group compared to the CON group ( $\mathbf{F i g}$. $\mathbf{5 a - c}$ ). Meanwhile, miR-21 and miR-29b levels were upregulated $\sim 1.79$-fold $(P=0.0292)$ and $\sim 1.67$-fold $(P=0.0291)$, respectively, in the hearts of mice in the OTR/down after treadmill running compared to those of mice in the NT group, but miR-17-3p expression remained unchanged. Interestingly, compared to the OTR group, the OTR/down group exhibited responses to its training protocol that were similar to those of the OTR group, and the mice exhibited upregulated expression of miR-21 ( 1.89-fold; $P=0.0233$ ), miR-17-3p ( 1.73-fold; $\mathrm{P}=0.0084)$ and miR-29b ( 1.69-fold; $\mathrm{P}=0.0225)$. Notably, unlike the effects on the LV myocardium, overtraining and aerobic training did not seem to affect the expression of these miRNAs in the RV myocardium ( $\mathbf{F i g . 5 d - f ) . ~}$

\section{Discussion}

Various molecules in the heart have been studied by researchers to better understand the underlying mechanisms associated with overtraining during aerobic exercise. In our study, among the three
OT protocols, only the OTR/down protocol affected cardiac-related miRNA levels, but the miR-208a level did not change significantly. These changes in miRNA expression may potentially indicate the occurrence of overtraining. Excessive treadmill training produces distinctly different cardiac-related miRNA profiles in the right and left ventricles.

Endurance training plays a very important role in physiological cardiac remodelling, which is closely related to the cellular, molecular and metabolic adaptation gene regulation mechanism and cellular signalling [26]. The expression of individual miRNAs, key small molecules that regulate gene expression, have been investigated in the left ventricle, which revealed the important and critical contributions of many of these miRNAs to acute/chronic exercise $[27,28]$. For example, the downregulation of miR-1, miR-133a and miR-133b in the LV of rats by aerobic training controls target mRNAs, such as Ras homologue gene family-A, cell division control protein 42, and Wolf-Hirschhorn syndrome candidate 2, which may contribute to physiological cardiac hypertrophy [29]. These mRNAs are also downregulated in pathological cardiac hypertrophy, and their common patterns of expression suggest their involvement in general programmes of cardiac hypertrophy [29]. In our study, although the downregulation of miR-1, miR-133a and miR-133b was not observed in the NT group, the OTR/down group showed the upregulated expression of these miRNAs. Moreover, past studies have proven that miR-1 overexpression disrupts myoganglion assembly [30] and increases susceptibility to atrioventricular block during myocardial ischaemia [31], suggesting pathological remodelling. Thus, our data suggest that the increase in miR-1 levels induced by the OTR/down protocol likely causes maladaptive remod- 
elling. The overexpression of miR-133a in the diabetic Akita heart decreases cardiac fibrosis, hypertrophy, and diabetic cardiomyopathy [32], and overexpression of miR-133b blocks the alteration in the gene expression pattern mediated by $\beta$-adrenergic receptor stimulation [33]. Notably, $\beta$-adrenaline sensitivity induced by oestrogen receptor regulation in women may play a special role in vascular changes [34-36]. More importantly, in humans, duration-independent LV assist device support leads to the decreased expression of miR-1, miR-133a, and miR-133b in patients with dilated cardiomyopathy, while the opposite result is observed in patients with ischaemic heart disease [37], suggesting that the activation of these miRNA signalling pathways serves as a potential repair mechanism for cardiac processes. In addition, inhibition of miR-206 expression aggravates ischaemia/reperfusion injury and prevents cardiac hypertrophy caused by overload [38]. Conversely, miR-206 overexpression in rats improves ischaemic injury-induced cardiomyocyte apoptosis by targeting tyrosine phosphatase 1B and thus exerts a protective effect [39], and overexpression of miR-206 causes cardiac hypertrophy in mice and protects them from ischaemia/ reperfusion injury [38]. Because exercise can mimic the effect of ischaemic preconditioning and may induce pressure or volume overload, our data likely suggest that increased miR-133a and miR$133 \mathrm{~b}$ levels are linked to cardioprotection/repair.

Moreover, the roles of miR-208a, miR-208b and miR-499, known as 'MyomiRs', have been identified in physiological models [40]. Heavy-load swimming training reduces the expression of miR-208a and miR-208b in the mouse heart and regulates the expression of hypertrophy/metabolism-related signalling molecules as an adaptation to physiological signals [40]. The levels of miR-208b and miR499 are upregulated in failing and hypertrophied human hearts [41] and in mouse models of pathological hypertrophy [42]. Upregulation of miR-499 accelerates the maladaptation to pressure overload through direct and indirect cumulative effects on the posttranslational modification of myocardial proteins [43]. Thus, changes in miR-208b and miR-499 expression induced by the OTR/ down protocol may be related to a pathological condition.

Moreover, the roles of miR-21, miR-17-3p and miR-29b have been studied in physiological models of cardiac hypertrophy [13]. These studies showed increased cardiac levels of miR-21, miR-17$3 p$ and miR-29b in swimming-trained mice, and this increase is involved in regulating cardiac hypertrophy $[44,45]$, myocyte proliferation [45], cardiac apoptosis [45], fibrosis [46] and cardioprotection [45]. More importantly, wheel exercise or treadmill running also increases mouse ventricular miR-17-3p levels [45]. In our study, the OTR/down group also presented increased miR-21, miR$17-3 p$ and miR-29b levels, likely suggesting a protective effect of these miRNAs on cardiac adverse remodelling.

Exercise exerts a longer term effect on the structure and function of the RV [47]. Previous studies have suggested that the increases in load in the RV are greater than those in the LV and that the contraction force may not be sufficient to maintain the output required for continuous high-intensity exercise, which can place the RV under greater stress $[47,48]$. For example, the release of brain natriuretic peptide (a marker of myocardial strain) is correlated with RV dysfunction only in prolonged endurance training [49]. In addition, the degree of RV remodelling in endurance ath- letes caused by exercise appears to be slightly greater than that of $\mathrm{LV}$ remodelling. Therefore, some people propose that the $\mathrm{RV}$ is the key factor that determines aerobic capacity [47]. Currently, few investigations on miRNAs and physiological RV remodelling have been performed. In our study, the levels of selected miRNAs did not change in the RV, which encircles the common myocardial fibre, in response to the NT and three OT protocols. According to a previous study, long-term aerobic treadmill exercise training promotes distinct metabolic adaptations in the RV and LV of female SpragueDawley rats [50]. This result suggests that the adaptive changes in the LV and RV myocardium after exercise appear to be sex-specific. Given these differences between the RV and LV, the two chambers likely display different miRNA responses under conditions of exercise training. Moreover, while RV-specific expression profiles of miRNAs have been identified, further studies are needed to determine whether exercise influences the expression of these miRNAs [51].

In addition, the lack of recovery and the continued imbalance of excess demand may trigger a series of unfavourable conditions, which may lead to decreased performance or weight. In the present study, although the weight of mice in the three OT groups did not change, their performance showed a significant decrease, which is a key sign of OT [9]. These changes were mainly detected in the LV, including cardiac fibrosis and pathological hypertrophy development [10]. Thus, the rest and recovery characteristics of the OTR/down protocol may adequately elicit an overtraining response. At present, the overtraining continuum has been modified. NFOR and OT have the same spectrum of responses that potentially lead to OTS. Because physiological and pathological redundancy plays a key role in the different cardiac stimuli or stresses, these responses are likely integrated. More importantly, the degree of participation of different muscle groups is different in different exercise methods. Previous studies have shown increased levels of proinflammatory cytokines in the serum, hypothalamus, muscle and liver of mice subjected to the OTR/down regimen [51, 52]. Additionally, more fibre damage, soreness and decreased performance were reported, which seem to be possible factors contributing to the differential expression of miRNAs among the three OT groups [53]. Although OT alters cardiac-related miRNA levels, the mechanisms by which OT increases the expression of these miRNAs should be further studied. Each of these mechanisms for individual miRNAs may affect cardiac remodelling, depending on the intensity, duration and frequency with which the signal appears. Defining the specific mechanism that mediates OT is an important starting point that can be used to develop optimized exercise interventions to improve body health.

\section{Conclusions}

Taken together, OT generally affects the expression of some key miRNAs in the LV and that changes in individual miRNAs may cause either adaptive or maladaptive remodelling with OT. In addition, the LV and RV were differently affected by OT. 


\section{Acknowledgements}

We thank Xin peng ZHANG for helpful comments on the manuscript. We would also like to thank Jin zhi WANG for his help with data collection and organization.

\section{Funding}

The authors acknowledge that this work was supported by the Research Special Fund for PLA University of Science and Technology (KYJYZLXY1603-9, 40). The Research Innovation Program for College Graduates of Jiangsu Province (KYCX19_1471). The Research Fund for Postgraduate in Military (KYJXJQJY2001). The Natural Science Foundation of Jiangsu Province (2021).

\section{Conflict of Interest}

The authors declare that they have no conflict of interest.

\section{References}

[1] Hawley JA, Hargreaves M, Joyner MJ et al. Integrative biology of exercise. Cell 2014; 159: 738-749. doi:10.1016/j.cell.2014.10.029

[2] Seo DY, Kwak HB, Kim AH et al. Cardiac adaptation to exercise training in health and disease. Pflugers Arch 2020; 472: 155-168. doi:10.1007| s00424-019-02266-3

[3] Bernardo BC, Ooi JYY, Weeks KL et al. Understanding key mechanisms of exercise-induced cardiac protection to mitigate disease: current knowledge and emerging concepts. Physiol Rev 2018; 98: 419-475. doi:10.1152/physrev.00043.2016

[4] Wilson MG, Ellison GM, Cable NT. Basic science behind the cardiovascular benefits of exercise. Br J Sports Med 2016; 50: 93-99. doi:10.1136/bjsports-2014-306596rep

[5] Morganroth J, Maron B], Henry WL et al. Comparative left ventricular dimensions in trained athletes. Ann Intern Med 1975; 82: 521-524. doi:10.7326/0003-4819-82-4-521

[6] Ellison GM, Waring CD, Vicinanza $C$ et al. Physiological cardiac remodelling in response to endurance exercise training: Cellular and molecular mechanisms. Heart 2012; 98: 5-10. doi:10.1136/ heartjnl-2011-300639

[7] Nakamura M, Sadoshima J. Mechanisms of physiological and pathological cardiac hypertrophy. Nat Rev Cardiol 2018; 15: 387-407. doi:10.1038/s41569-018-0007-y

[8] Fry AC, Kraemer W]. Resistance exercise overtraining and overreaching. Neuroendocrine responses. Sports Med 1997; 23: 106-129. doi:10.2165/00007256-199723020-00004

[9] Meeusen R, Duclos M, Foster C et al. Prevention, diagnosis, and treatment of the overtraining syndrome: joint consensus statement of the European College of Sport Science and the American College of Sports Medicine. Med Sci Sports Exerc 2013; 45: 186-205. doi:10.1249/MSS.0b013e318279a10a

[10] da Rocha AL, Teixeira GR, Pinto AP et al. Excessive training induces molecular signs of pathologic cardiac hypertrophy. J Cell Physiol 2018; 233: 8850-8861. doi:10.1002/jcp.26799

[11] Fernandes T, Soci UP, Oliveira EM. Eccentric and concentric cardiac hypertrophy induced by exercise training: microRNAs and molecular determinants. Braz J Med Biol Res 2011; 44: 836-847. doi:10.1590/ s0100-879x2011007500112
[12] Fernandes T, Barauna VG, Negrao CE et al. Aerobic exercise training promotes physiological cardiac remodeling involving a set of microRNAs. Am J Physiol Heart Circ Physiol 2015; 309: H543-H552. doi:10.1152/ajpheart.00899.2014

[13] Schuttler D, Clauss S, Weckbach LT et al. Molecular mechanisms of cardiac remodeling and regeneration in physical exercise. Cells 2019; 8: 1128 . doi: $10.3390 /$ cells 8101128

[14] Chistiakov DA, Orekhov AN, Bobryshev YV. Cardiac-specific miRNA in cardiogenesis, heart function, and cardiac pathology (with focus on myocardial infarction). J Mol Cell Cardiol 2016; 94: 107-121. doi:10.1016/j.yjmcc.2016.03.015

[15] Lindman BR, Dweck MR, Lancellotti P et al. Management of asymptomatic severe aortic stenosis: Evolving Concepts in timing of valve replacement. JACC Cardiovasc Imaging 2020; 13: 481-493. doi:10.1016/j.jcmg.2019.01.036

[16] Rodrigues JC, Amadu AM, Dastidar AG et al. Comprehensive characterisation of hypertensive heart disease left ventricular phenotypes. Heart 2016; 102: 1671-1679. doi:10.1136/ heartjnl-2016-309576

[17] Treibel TA, Lopez B, Gonzalez A et al. Reappraising myocardial fibrosis in severe aortic stenosis: an invasive and non-invasive study in 133 patients. Eur Heart J 2018; 39: 699-709. doi:10.1093/eurheartj/ ehx353

[18] Ravassa S, Lopez B, Querejeta R et al. Phenotyping of myocardial fibrosis in hypertensive patients with heart failure. Influence on clinical outcome. J Hypertens 2017; 35: 853-861. doi:10.1097/ HJH.0000000000001258

[19] Carabello BA. Aortic stenosis: from pressure overload to heart failure. Heart Fail Clin 2006; 2: 435-442. doi:10.1016/j.hfc.2006.11.001

[20] Batkai S, Bar C, Thum T. MicroRNAs in right ventricular remodelling. Cardiovasc Res 2017; 113: 1433-1440. doi:10.1093/cvr/cvx153

[21] Pereira BC, Filho LA, Alves GF et al. A new overtraining protocol for mice based on downhill running sessions. Clin Exp Pharmacol Physiol 2012; 39: 793-798. doi:10.1111/j.1440-1681.2012.05728.x

[22] Harriss D], MacSween A, Atkinson G. Ethical standards in sport and exercise science research: 2020 update. Int J Sports Med 2019; 40: 813-817. doi:10.1055/a-1015-3123

[23] Kuipers H, Verstappen FT, Keizer HA et al. Variability of aerobic performance in the laboratory and its physiologic correlates. Int J Sports Med 1985; 6: 197-201. doi:10.1055/s-2008-1025839

[24] Turgeman T, Hagai Y, Huebner $\mathrm{K}$ et al. Prevention of muscle fibrosis and improvement in muscle performance in the $\mathrm{mdx}$ mouse by halofuginone. Neuromuscul Disord 2008; 18: 857-868. doi:10.1016/j. nmd.2008.06.386

[25] Schneider CA, Rasband WS, Eliceiri KW. NIH Image to Image]: 25 years of image analysis. Nat Methods 2012; 9: 671-675. doi:10.1038/ nmeth.2089

[26] Vega RB, Konhilas JP, Kelly DP et al. Molecular mechanisms underlying cardiac adaptation to exercise. Cell Metab 2017; 25: 1012-1026. doi:10.1016/j.cmet.2017.04.025

[27] Wang L, Lv Y, Li G et al. MicroRNAs in heart and circulation during physical exercise. J Sport Health Sci 2018; 7: 433-441. doi:10.1016/j. jshs.2018.09.008

[28] Domanska-Senderowska D, Laguette MN, Jegier A et al. MicroRNA profile and adaptive response to exercise training: a review. int $\mathrm{j}$ Sports Med 2019; 40: 227-235. doi:10.1055/a-0824-4813

[29] Soci UP, Fernandes T, Hashimoto NY et al. MicroRNAs 29 are involved in the improvement of ventricular compliance promoted by aerobic exercise training in rats. Physiol Genomics 2011; 43: 665-673. doi:10.1152/physiolgenomics.00145.2010

[30] Ai ], Zhang R, Gao X et al. Overexpression of microRNA-1 impairs cardiac contractile function by damaging sarcomere assembly. Cardiovasc Res 2012; 95: 385-393. doi:10.1093/cvr/cvs196 
[31] Zhang Y, Sun L, Zhang Y et al. Overexpression of microRNA-1 causes atrioventricular block in rodents. Int J Biol Sci 2013; 9: 455-462. doi:10.7150/ijbs.4630

[32] Kambis TN, Shahshahan HR, Kar S et al. Transgenic expression of miR-133a in the diabetic akita heart prevents cardiac remodeling and cardiomyopathy. Front Cardiovasc Med 2019; 6: 45. doi:10.3389/ fcrm.2019.00045

[33] Sucharov C, Bristow MR, Port JD. miRNA expression in the failing human heart: functional correlates. J Mol Cell Cardiol 2008; 45: 185-192. doi:10.1016/j.yjmcc.2008.04.014

[34] Hart EC, Charkoudian N, Wallin BG et al. Sex and ageing differences in resting arterial pressure regulation: the role of the beta-adrenergic receptors. J Physiol 2011; 589: 5285-5297. doi:10.1113/jphysiol.2011.212753

[35] Murphy E. Estrogen signaling and cardiovascular disease. Circ Res 2011; 109: 687-696. doi:10.1161/CIRCRESAHA.110.236687

[36] Dworatzek E, Mahmoodzadeh S, Schubert C et al. Sex differences in exercise-induced physiological myocardial hypertrophy are modulated by oestrogen receptor beta. Cardiovasc Res 2014; 102: 418-428. doi: $10.1093 / c v r / c v u 065$

[37] Schipper ME, van Kuik J, de Jonge $\mathrm{N}$ et al. Changes in regulatory microRNA expression in myocardium of heart failure patients on left ventricular assist device support. J Heart Lung Transplant 2008; 27: 1282-1285. doi:10.1016/j.healun.2008.09.005

[38] Yang Y, Del Re DP, Nakano N et al. miR-206 mediates YAP-induced cardiac hypertrophy and survival. Circ Res 2015; 117: 891-904. doi:10.1161/CIRCRESAHA.115.306624

[39] Yan Y, Dang H, Zhang $X$ et al. The protective role of MiR-206 in regulating cardiomyocytes apoptosis induced by ischemic injury by targeting PTP1B. Biosci Rep 2020; 40: BSR20191000. doi:10.1042/ BSR20191000

[40] Soci UPR, Fernandes T, Barauna VG et al. Epigenetic control of exercise training-induced cardiac hypertrophy by miR-208. Clin Sci (Lond) 2016; 130: 2005-2015. doi:10.1042/CS20160480

[41] Satoh M, Minami Y, Takahashi Y et al. Expression of microRNA-208 is associated with adverse clinical outcomes in human dilated cardiomyopathy. J Card Fail 2010; 16: 404-410. doi:10.1016/j.cardfail.2010.01.002

[42] Gan Z, Rumsey J, Hazen BC et al. Nuclear receptor/microRNA circuitry links muscle fiber type to energy metabolism. J Clin Invest 2013; 123: 2564-2575. doi:10.1172/JCI67652
[43] Matkovich S], Hu Y, Eschenbacher WH et al. Direct and indirect involvement of microRNA-499 in clinical and experimental cardiomyopathy. Circ Res 2012; 111: 521-531. doi:10.1161/CIRCRESAHA. 112.265736

[44] Ma Z, Qi J, Meng S et al. Swimming exercise training-induced left ventricular hypertrophy involves microRNAs and synergistic regulation of the PI3K/AKT/mTOR signaling pathway. Eur J Appl Physiol 2013; 113: 2473-2486. doi:10.1007/s00421-013-2685-9

[45] Shi ], Bei Y, Kong X et al. miR-17-3p contributes to exercise-induced cardiac growth and protects against myocardial ischemia-reperfusion injury. Theranostics 2017; 7: 664-676. doi:10.7150/thno.15162

[46] Chaturvedi P, Kalani A, Medina I et al. Cardiosome mediated regulation of MMP9 in diabetic heart: role of mir29b and mir455 in exercise. J Cell Mol Med 2015; 19: 2153-2161. doi:10.1111/jcmm. 12589

[47] Chivulescu M, Haugaa K, Lie OH et al. Right ventricular remodeling in athletes and in arrhythmogenic cardiomyopathy. Scand Cardiovasc J 2018; 52: 13-19. doi:10.1080/14017431.2017.1416158

[48] D'Andrea A, La Gerche A, Golia E et al. Right heart structural and functional remodeling in athletes. Echocardiography 2015; 32: S11-S22. doi:10.1111/echo.12226

[49] La Gerche A, Burns AT, Mooney DJ et al. Exercise-induced right ventricular dysfunction and structural remodelling in endurance athletes. Eur Heart J 2012; 33: 998-1006. doi:10.1093/eurheartj/ ehr397

[50] Nogueira-Ferreira R, Ferreira R, Padrao Al et al. One year of exercise training promotes distinct adaptations in right and left ventricle of female Sprague-Dawley rats. J Physiol Biochem 2019; 75: 561-572. doi:10.1007/s13105-019-00705-4

[51] Aoi W. Frontier impact of microRNAs in skeletal muscle research: a future perspective. Front Physiol 2014; 5: 495. doi:10.3389/ fphys.2014.00495

[52] da Rocha AL, Pereira BC, Teixeira GR et al. Treadmill slope modulates inflammation, fiber type composition, androgen, and glucocorticoid receptors in the skeletal muscle of overtrained mice. Front Immunol 2017; 8: 1378. doi:10.3389/fimmu.2017.01378

[53] Davis JM, Murphy EA, Carmichael MD et al. Curcumin effects on inflammation and performance recovery following eccentric exercise-induced muscle damage. Am J Physiol Regul Integr Comp Physiol 2007; 292: R2168-R2173. doi:10.1152/ajpregu.00858.2006 